\title{
A gestão ambiental, novo desafio para a psicologia do desenvolvimento sustentável ${ }^{1}$
}

\author{
Enric Pol \\ Universidade de Barcelona
}

\begin{abstract}
Resumo
Todo projeto industrial, desenvolvimento urbano ou oferta de serviço implica modificações em seu entorno, um impacto ambiental que pode afetar mais do que o local físico. Essas mudanças podem ser gestionadas positivamente, para minimizar seu impacto, ou podem ser ignoradas, deixando que o meio alterado (físico e social) siga seu curso, normalmente em um processo de degradação cada vez mais acelerado. A forma atual de entender a gestão ambiental remete ao conjunto de ações preventivas e paliativas para minimizar os efeitos ambientais da atividade humana. A gestão ambiental é antes de tudo gestão do comportamento humano, tanto dentro como fora das organizações. A pergunta que devemos nos fazer é se a Psicologia Ambiental tem suficiente bagagem - ou se está disposta a desenvolvê-la - para fazer frente ao desafio e assumir a responsabilidade de estar presente na gestão ambiental. Este artigo revisa algumas novas propostas nesse campo.
\end{abstract}

Palavras-chave: gestão ambiental; avaliação do impacto ambiental; efeito nimby; participação; psicologia ambiental

\begin{abstract}
Environmental management, new challenge for a psychology of sustainable development. Any industrial project, urban development or service provision leads to changes in its setting - an environmental impact that can extend well beyond the immediate site. These changes can be managed positively, in an attempt to mitigate this impact, or they can be ignored allowing the transformed environment (in its physical and social aspects) to continue along its path of change, often in an increasingly rapid process of degradation. Environmental management is today understood to include preventive and palliative actions aimed at minimizing the environmental impact of human activity. Environmental management is above all concerned with management of human behavior both within and outside organizations. The question that should be posed therefore is whether Environmental Psychology has sufficient experience - or whether is willing to develop it - to face the challenge and take on the responsibilities of being present within environmental management. This paper examines some new propositions in the field.
\end{abstract}

Key words: environmental management; environmental impact assessment; nimby effect; participation; environmental psychology

$\mathrm{U}$ ma psicologia ambiental com vocação de contribuir para o progresso da sociedade, desenvolvendo conhecimento básico, aplicável e aplicado, requer assumir os desafios sociais de cada momento histórico. Se não, corremos o risco de nos tornarmos um "fóssil" acadêmico ou um "luxo para os ricos que os ricos não nos pedem” (Pol, 1993, 1998). Os desafios que a Psicologia Ambiental tem a enfrentar na sociedade atual apresentam pelo menos duas dimensões: uma de intervenção (gerar mudanças no meio ambiente) e outra de gestão (tomar decisões a partir de uma escala de valores implícita ou explícita), ambas com base nos parâmetros da sustentabilidade como novo valor social positivo; valor necessário para a sobrevivência do planeta e da espécie humana, segundo se depreende dos debates sociais e das propostas das Nações Unidas no Informe Brundtland (1987) e das Conferencias Rio’92 e Johanesburgo’02.
A intervenção ambiental apresenta, por sua vez, duas formas. Por um lado, o que sincreticamente se pode chamar de criação de lugares, um componente da tradição mais característica da Psicologia Ambiental Arquitetônica. Por outro, encontra-se a planificação e o projeto de infra-estruturas e serviços, menos comum na literatura da Psicologia Ambiental. Ambas estão relacionadas ao bem-estar das pessoas e à sua qualidade de vida. A situação nova, em relação aos planejamentos clássicos da Psicologia Ambiental, é que a "unidade de medida" da qualidade de um projeto (em qualquer uma das duas acepções mencionadas) já não é somente a pessoa, mas sim a pessoa em um ambiente sustentável.

Ainda que o objetivo último de um desenvolvimento sustentável possa ser comum a toda a humanidade, os objetivos imediatos, as estratégias e a orientação das ações para alcançá-los podem (e devem) ser específicos de cada lugar. 
Estes podem até mesmo chegar a ser opostos àquele, especialmente se consideramos a situação nos países desenvolvidos do ocidente e a dos países empobrecidos. Por isso mesmo, não faltam razoáveis visões críticas do conceito de desenvolvimento sustentável. Assim, por exemplo, Allende (1995) assinala que o Informe Brundtland (1987) oferece somente soluções tecnocratas que, a médio prazo, não são sustentáveis (Olson, 1995). Cobb (1995) afirma que o desenvolvimento sustentável deveria preservar a auto-suficiência das diversas regiões. A solução não consiste em gerar recursos por meio do crescimento, mas sim por intermédio da redistribuição de recursos e tecnologia de maneira mais eqüitativa, respeitando as formas locais de produção, que em grande medida se ajustam à capacidade do ecossistema (MartínezAlier, 1992). Milbrath $(1986,1995)$ destaca que a raiz do problema se encontra no atual sistema de crenças do paradigma social dominante. Corson (1995) recomenda que os programas de conscientização ambiental sejam intensificados, ao mesmo tempo em que se busca reduzir as injustiças sociais e políticas.

Neste trabalho definiremos alguns conceitos centrais de intervenção e gestão ambientais e sintetizaremos algumas contribuições fundamentais para tratar das dimensões comportamentais implícitas ou explícitas nos instrumentos mais típicos desse âmbito, que afetam tanto as empresas como as administrações públicas.

A Psicologia Ambiental clássica esteve centrada quase exclusivamente no comportamento da pessoa como indivíduo, e pouco no conhecimento que possa ser útil para a tomada de decisões na gestão, como já afirmavam Stern e Oskamp (1987). Nos últimos anos, entretanto, começou a haver um crescente enfoque empírico e conceitual nessa direção. Uma comprovação desse fato está no número de artigos e números monográficos em distintas revistas internacionais de Psicologia, entre elas (embora não sejam as únicas) Environment and Behavior, Journal of Environmental Psychology e Journal of Social Issues. Esta última publicou quatro números temáticos nos últimos dez anos (1992, 1994, 1995, 2000), com participação de autores que se destacam como os de maior visibilidade sobre o tema, como Oskamp, Stern, Schultz, ou McKenzie-Mohr, entre outros. Também não é por acaso que no ano 2002 apareceram quase simultaneamente pelo menos seis títulos com referência ou contribuições explícitas ao desenvolvimento sustentável, à gestão e promoção ambientais e à cidade sustentável (Bechtel \& Churchman, 2002; García-Mira, Sabucedo \& Romay, 2002; Moser, Ratiu \& Fleury-Bahi, 2002; Pol, 2002a, 2002b; Schultz \& Schmuck, 2002).

\section{Alguns conceitos chaves}

Nesta seção definiremos alguns conceitos-chave que caracterizam o âmbito da intervenção e gestão ambientais e que são a base das aplicações que veremos nas seções a seguir.

Como sintetizado na Figura 1, toda intervenção provoca impactos ambientais, que têm, pelo menos, uma dimensão física e uma dimensão social que altera (positiva ou negativamente) o ecossistema, o comportamento e o bem-estar das pessoas. Impactos, efeitos e mudanças que se gestionam por ação ou por omissão.

Entendemos por gestão ambiental (Moreno \& Pol, 1999) aquela que incorpora os valores do desenvolvimento sustentável na organização social e nas metas corporativas da empresa e da administração pública. Integra políticas, programas e práticas relativas ao meio ambiente, em um processo contínuo de melhoria da gestão.

A gestão ambiental se centra principalmente nos recursos. Pressupõe escolher entre alternativas (que não são somente tecnológicas) e criar as condições para que aconteça o que se pretende que aconteça. Deve controlar e assegurar o cumprimento da lei ou normas que estão estabelecidas e regulam os comportamentos das pessoas, das sociedades, das empresas, das formas de produção e seus efeitos. Porém também deve levar em consideração as pessoas e seu comportamento.

\section{Dimensões psicológicas nos instrumentos típicos da intervenção e gestão ambientais nas organizações}

O universo da intervenção e gestão ambientais constantemente gera novas figuras e novos instrumentos, que sempre acabam incidindo no comportamento das pessoas. Uma das características desse setor é que boa parte deles surge a partir de recomendações, normativas e regulamentos de organismos internacionais como Nações Unidas ou International Standards Organization (ISO), além das legislações de cada país. Focalizaremos aqui alguns aspectos típicos e complementares que se aplicam a três níveis distintos: sobre a organização em funcionamento, sobre um projeto antes de sua construção e sobre um produto (outros aspectos serão apresentados mais adiante, na seção sobre administração pública).

Figuras e instrumentos de gestão ambiental para organizações em funcionamento

Boa parte das figuras legislativas e instrumentos para a gestão está orientada à mudança ambiental nas organizações já existentes. Eles têm por objetivo estimular e/ou facilitar uma mudança de estilo nos modos de fazer, uma modificação de comportamento da organização e seus membros e obter certificações ambientais que, teoricamente, situarão a organização em melhor posição em um mercado competitivo. A seguir, trataremos da implantação de sistemas de gestão ambiental e dos processos de auditorias ambientais usados para seu controle ou verificação (conforme o caso).

Sistema de gestão ambiental. A norma de sistema de gestão ambiental (SGA, ou EMS, Environmental Management System) mais conhecida internacionalmente é a ISO 14000, embora existam países que possuem suas próprias legislações. A ISO 14000, de 1996, define SGA como aquela parte do sistema geral de gestão da organização que compreende a estrutura organizativa, as responsabilidades, as práticas, os 


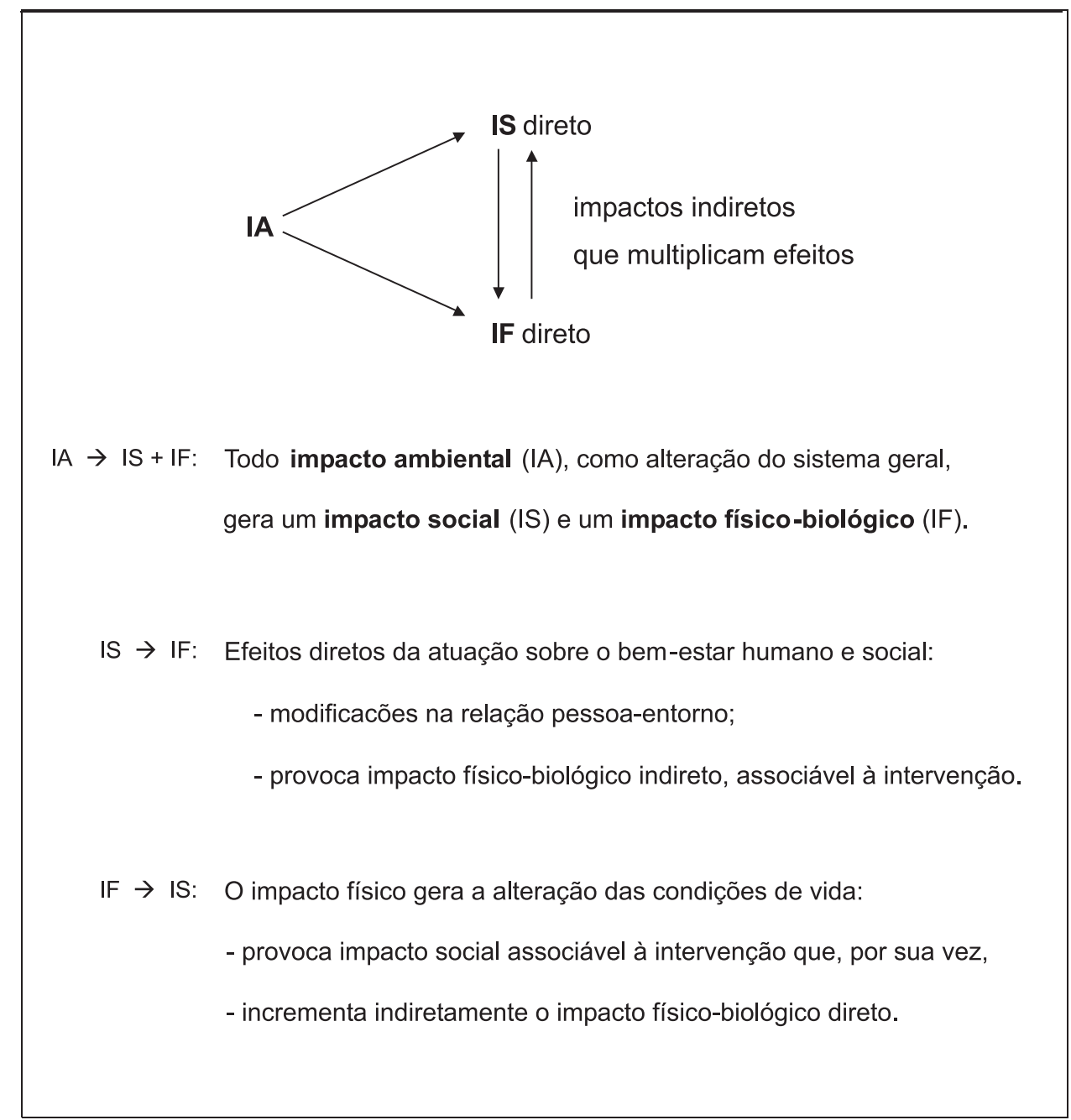

Figura 1. Impacto ambiental - impacto social - impacto ecológico (Fonte: Pol, 2001).

procedimentos, os processos e os recursos para determinar e executar sua política ambiental. Na implantação de um SGA se distinguem cinco etapas:

1. Conscientizar: nesta etapa é especialmente importante envolver a alta direção.

2. Comprometer: a formulação da política ambiental, em forma de declaração, deverá ser pública e divulgada.

3. Organizar: a partir de um diagnóstico ambiental inicial, as tecnologias e os processos são revisados; e também a adequação do organograma e das mudanças necessárias; estabelecem-se objetivos ambientais; e redige-se o manual de gestão ambiental e os manuais de boas práticas.

4. Implementar o SGA: na implementação deve-se estabelecer os sistemas de controle de operações, de gestão e de registros.
5. Verificar e revisar: o SGA contempla um programa de auditorias ambientais, de sistemas de informação, comunicação, informes, marketing, etc., como formas que permitam a verificação sistemática do correto funcionamento de acordo com o estabelecido na política ambiental da organização.

Uma vez comprovada a eficácia do sistema e o cumprimento dos requisitos estabelecidos na norma, procede-se à solicitação de uma certificação do sistema.

Em cada uma das fases de implantação de um SGA existe uma série de aspectos psicológicos implicados: valores, atitudes e condutas; formação ambiental, cultura organizacional e gestão das mudanças nas organizações, condições ambientais de trabalho como elementos de conforto, de segurança, de oportunidade ou facilitadoras dos comportamentos desejados, relações de grupo, influência social, comunicação e liderança. 
Mesmo que a implantação de SGA signifique um progresso em direção a um modelo mais sustentável, ela não está isenta de aspectos críticos. Como destacado por Kirkland e Thompson (1999), existe uma importante defasagem entre a teoria e a prática. O principal obstáculo para a adoção de um SGA é a própria organização (Moxen \& Strachan, 2000) e sua resistência às mudanças internas e às mudanças em suas relações com a comunidade e parceiros externos (Jørgensen, 2000). Para Hillary (1999), a falta de formação suficiente e eficiente dos membros da organização; a insuficiente transmissão dos objetivos e dos valores ambientais aos próprios gestores; a percepção de que a própria organização não gera impactos ambientais importantes e de que os clientes não valoram uma melhora no seu comportamento ambiental são um obstáculo à adoção de um SGA que alcance além do estritamente formal.

Auditoria ambiental. A auditoria ambiental (AA) é um instrumento de verificação que permite a avaliação sistemática documentada, periódica e objetiva da eficácia da organização, do SGA e dos procedimentos planejados para a proteção do meio ambiente. Seu objetivo é facilitar à direção da organização (empresa ou administração pública) a informação precisa para o controle dos trabalhos, comportamentos e/ou ações que possam ocasionar efeitos sobre o meio ambiente, e avaliar sua adequação à política ambiental preestabelecida.

Aspectos organizacionais, sociais e comportamentais a considerar. Os manuais profissionais para a realização de auditorias ambientais (e.g., Chermisinoff \& Chermisinoff, 1993; Harrison, 1995) incluem questionários, checklists, fichas de observação, etc., que costumam ser muito exaustivos nas dimensões tecnológicas, porém insuficientes nas dimensões organizacionais. Sincreticamente, a partir de uma ótica psicológica, Moreno (1995) considera os seguintes âmbitos:

- a estrutura organizacional e tipo de gestão, que inclui entre outros aspectos, o estilo de relações, o nível de participação dos trabalhadores nas decisões organizacionais;

- estilo de liderança e processos de tomada de decisões, que inclui o clima organizacional, a gestão das mudanças organizacionais e as motivações relacionadas às mudanças;

- a política ambiental e os responsáveis ambientais, que trata de como se estabelece a política ambiental e de quem decide criá-la, recursos materiais e recursos humanos dedicados a temas ambientais, descrição de tarefas e condições de trabalho;

- motivação e comunicação, envolvendo os sistemas de reconhecimento das contribuições dos empregados em relação a temas ambientais, sistemas internos de promoção, comunicação interna e externa.

\section{Figuras e instrumentos de gestão ambiental para projetos antes de sua construção}

Uma das principais dimensões da gestão ambiental é a preventiva, que compreende a avaliação prospectiva de projetos mediante o Estudo de Impacto Ambiental (EIA).
O EIA é o instrumento mais antigo para a gestão preventiva do meio ambiente (ver, em relação aos EUA, National Environmental Policy Act / NEPA, em United StatesEnvironmental Protection Agency, 1969; ou, relativas à Europa, as diretivas 85/337/CEE e 97/11/CE, em European Commission, 1985, 1997). Ele compreende a avaliação dos efeitos que uma instalação industrial ou um serviço pode causar sobre o meio ecológico, o bem-estar humano e o patrimônio cultural e, em cada caso, recomendar medidas de correção, prevenção ou compensação. O EIA é realizado sobre o projeto de uma indústria, de uma infra-estrutura ou de um serviço, antes de ser autorizada sua construção.

É preciso distinguir entre Estudo de Impacto Ambiental (EIA) e Relatório de Impacto Ambiental (RIMA). O EIA é o processo de decisão que os organismos oficiais competentes realizam a partir do projeto e do RIMA que deve sempre acompanhá-lo. O Relatório de Impacto Ambiental (RIMA) é o documento resultante do trabalho de análise, detecção e descrição dos efeitos previsíveis vinculados ou vinculáveis ao projeto da instalação ou serviço que se pretende construir, e deve contemplar propostas para a minimização de tais efeitos.

A partir do projeto, do RIMA e das alegações que a população possa apresentar durante o período de audiências públicas prévias, o órgão competente da administração pública prepara uma Declaração de Impacto, em que aprova ou indefere a autorização para a construção do projeto, incrementando, se necessário, as medidas preventivas ou as compensatórias. Conforme a maioria das legislações específicas de cada país, devem ser levados em consideração tanto os impactos físicos como os sociais.

O Comitê Interorganizacional para Diretrizes e Princípios para a Avaliação de Impacto Social (ICGPSIA, 1995) dos EUA define o impacto social como as conseqüências para as populações humanas de qualquer ação, pública ou privada, que altere a maneira como as pessoas vivem, trabalham, atuam, se relacionam com os demais, se organizam para satisfazer suas necessidades e, em geral, como se comportam como membros da sociedade. Para avaliar estes aspectos, o ICGPSIA (1995) considera que um estudo de impacto social deve contemplar:

- o uso do solo e dos recursos disponíveis para a comunidade;

- a existência ou não de serviços essenciais e a possibilidade de serem alterados;

- os efeitos no emprego, da distribuição dos custos e dos benefícios;

- as relações sociais, a qualidade de vida; e

- os significados subjetivos dos espaços, dos recursos e dos efeitos que a intervenção possa ter.

A partir da Psicologia Ambiental foram desenvolvidos alguns métodos para detecção e valoração de impactos sociais. Um deles é o DIS/BCN (Pol \& Moreno, 1994, 2002). Entre outros aspectos, o método contempla a necessidade de realizar uma valoração histórica e prospectiva da evolução social de lugar; considerar os efeitos na vertebração sociocultural 
(que inclui a organização social formal e informal) desde suas dimensões funcional e simbólica; e a informação, aceitação ou rejeição do projeto por parte da população. Esse método enfatiza a necessidade de um diagnóstico social inicial, como referência para a estimação de impactos sociais. Consiste de um roteiro para realizar o inventario social inicial e protocolos para a detecção de impactos com base em checklists versáteis e de fácil aplicação.

Outros métodos envolvem sistemas informatizados de simulação, como o SIMPACT (Huston \& DeSouza, 1980), ou propõem sistemas de quantificação padronizada dos impactos, como os chamados de orientação numérica, por exemplo, os do Instituto Battelle-Columbus (ver Battelle-Columbus Laboratories, 1972). Alguns utilizam estratégias combinadas, como os de Larry W. Canter (1977/1997). Torgerson (1980) utiliza estratégias participativas e Freudenberg (1986) enfatiza a necessidade de considerar as redes sociais na detecção de impactos. Taylor e Bryan (1990) destacam a conveniência de focalizar os problemas sociais já existentes e os que a nova intervenção pode gerar. O centro de referência europeu para os EIAs é o EIA Center da Universidade de Manchester, dirigido pelos professores $\mathrm{N}$. Lee e Ch. Wood, que publica o EIA Newsletter. No trabalho de Moreno e Pol (2002) pode-se encontrar ampla revisão de metodologias para a detecção de impactos sociais.

\section{A prevenção ambiental sobre um produto: a Análise do Ciclo de Vida}

A Análise do Ciclo de Vida (ACV, ou Life Cycle Analysis / LCA) é um instrumento de gestão ambiental centrado sobre um produto concreto, e não sobre a atividade global da organização. Sua finalidade é avaliar e reduzir, desde a fase de projeto, os impactos ambientais associados e associáveis ao produto. Além de um melhor comportamento ambiental da organização produtora, uma das utilidades e/ou benefícios de submeter um produto a uma ACV é a possibilidade de obter o direito de usar uma ecoetiqueta. A ACV está regulada pela ISO-14040 e a regulação das ecoetiquetas (ou ecolabels) pela ISO-14020, além das leis específicas de cada país.

Com uma ACV se pretende: reduzir o consumo de matérias primas e os impactos associados a sua extração e transporte, substituindo-as (quando possível) por subprodutos de outros processos industriais ou reciclando produtos já gastos; reduzir ou otimizar o consumo de energia nas fases de produção industrial e de uso do produto; e diminuir o volume e toxicidade dos resíduos resultantes. Ainda que essa questão pareça ser eminentemente tecnológica, tem uma dimensão direta e indiretamente vinculada ao comportamento humano e social.

Por princípio, a ACV requer uma vontade, uma disposição ética (que implica valores, atitudes e comportamentos) por parte dos promotores, planejadores e gestores. Mas também compromete o comportamento específico do usuário.

Na ACV pode-se diferenciar dois ciclos: o ciclo de projeto e produção e o ciclo do uso e disposição (Rieradevall, Moreno, Serena \& Pol, 1996). No ciclo de projeto e produção deve-se levar em consideração as tendências do comportamento do usuário, como condicionantes do projeto; além do comportamento das pessoas que intervém no ciclo produtivo, como fator humano que pode provocar desvios dos comportamentos desejados, com conseqüências ambientais. No ciclo do uso, é fundamental prever não só os usos primários, para os quais o produto foi desenhado, mas também os usos secundários ou reutilizações, e as destinações finais que podem ser dadas por grupos específicos de usuários. Em ambos os casos, trata-se de análise e previsão de comportamentos.

Assim, ao planejar-se um novo produto é preciso conhecer quais são as tendências de utilização e reutilização habituais dos grupos-alvo (target groups) ao quais o produto se dirige. É necessário prever um catálogo de funções secundárias factíveis e de usos e destinos finais a evitar. A explicação das formas desejáveis de utilização e destinação final suscitam um problema de comunicação eficiente, também relacionado aos campos de conhecimento tradicionais da Psicologia.

\section{Dimensões psicológicas na intervenção e gestão ambientais nas administrações públicas}

As administrações públicas (AP) desempenham pelo menos três papéis distintos em intervenção e gestão ambientais. Elas são em si organizações que desempenham um comportamento ambiental. Como tal, as APs podem (ou devem) ter um sistema de gestão ambiental próprio que pode optar por ser reconhecido ou certificado (via ISO 14000, por exemplo). Além disso, as APs são os órgãos competentes para o controle das ações ambientais das empresas (concedem e controlam autorizações de funcionamento, estudos de impacto ambiental, concessão de certificações ambientais a empresas ou a produtos, etc.). Nestes dois primeiros papéis, são aplicáveis as mesmas figuras e instrumentos descritos na seção anterior, referente a organizações.

O terceiro papel que as APs desempenham é uma gestão ambiental própria em seus âmbitos de competência (estabelecimento de políticas ambientais em nível nacional, regional ou local, planificação territorial, gestão de espaços naturais, política energética e de gestão de resíduos, de transporte, aplicação de uma agenda 21 local, etc.). Essa gestão nem sempre é bem aceita pelos coletivos sociais, encontrando, às vezes, resistências e reactâncias (efeito NIMBY, por exemplo). Voltaremos a esses tópicos nas subseções a seguir.

\section{Planejamento, desenho e gestão urbanística}

Este é o âmbito mais desenvolvido e divulgado na Psicologia Ambiental tradicional. Na sociedade ocidental, as administrações públicas são as responsáveis básicas pelo planejamento, desenho e gestão dos espaços urbanos e dos usos territoriais. Devem se ocupar da manutenção da qualidade ambiental, dos níveis de serviços (gestionados diretamente ou por meio de concessionárias) e, em última instância, da qualidade de vida e satisfação residencial da população, em que a apropriação do espaço e os aspectos relacionais aparecem como elementos-chave (Amérigo, 2000; Bell, Greene, 
Fisher \& Baum, 1996; Fleury-Bahi, 1997; Moser, Ratiu \& Fleury-Bahi, 2002; Pol, 1996, no prelo; Pol \& Valera, 1999). Mas as APs também são as principais promotoras de mudanças, melhorias, inovações ou de estabelecer objetivos estratégicos comuns. Isso implica conhecer as diferentes situações que existem, a detecção de necessidades e tendências sociais, o funcionamento dos sistemas técnicos, mas também das tendências de comportamento dos cidadãos que permitam que estes sistemas técnicos cumpram a função para a qual foram criados. Para cumprir com estes objetivos, as APs devem prover-se dos conceitos, instrumentos e recursos humanos adequados e suficientes. A Psicologia Ambiental pode (deve) contribuir para cada um desses objetivos.

Valera, Íñiguez, Pol e Llueca (1996) sintetizam um modelo para a intervenção ambiental que diferencia três fases:

- Fase I - Avaliação e seleção de necessidades manifestas e latentes, às quais a intervenção proposta deve dar resposta. A origem da iniciativa da intervenção e sua motivação real (melhorar a qualidade de vida, reabilitação de uma área, estimular a renovação de população, etc.), além da congruência do que se projeta com a realidade atual, sua aceitação ou rejeição por parte dos afetados, condicionarão inevitavelmente a avaliação. Isso implica considerar a memória histórica e a descrição atual do lugar e dos recursos disponíveis antes do projeto; a valoração atribuída pela população e o conhecimento de suas necessidades e expectativas.

- Fase II - Peritagem dos projetos: colaboração com os técnicos na concepção de projetos de intervenção e na previsão de seus efeitos. A peritagem de um projeto deve sempre realizar-se a partir de suas qualidades intrínsecas e em função da leitura social do lugar em que se projeta a intervenção. Isso implica dispor da informação sintetizada na fase I, ou seja, é necessário buscar ou criar essa informação, como ponto referencial de partida para a valoração, a fim de evitar desde o início do projeto que as decisões possam ter efeitos sociais e ambientais indesejáveis já que, uma vez finalizado, poderiam ter difícil correção por terem condicionado seu desenvolvimento. Uma vez finalizado o desenho do projeto, é necessário reavaliar os potenciais efeitos sociais e ambientais, para corrigir - se for preciso - o que se considere pouco desejável. Por isso é imprescindível a discussão conjunta com o cliente, os responsáveis políticos e os técnicos.

- Fase III - Valoração posterior à intervenção. A valoração se refere à estimação do valor de uma determinada intervenção. Mediante o uso dos métodos e das técnicas pertinentes a cada caso, avalia-se a consecução dos objetivos formulados na intervenção, sua adequação à população, a funcionalidade da intervenção, o grau de satisfação dos habitantes e o nível de identificação e apropriação que neles gerou, e o seu impacto socioambiental. Francescato, Weidemann e Anderson (1989) propõem métodos para avaliar a partir da perspectiva do usuário. As avaliações pós-ocupação (APO, ou POE, para Post Occupancy Evaluation) (Preiser, Rabinowitz e White, 1987) seriam um caso específico de avaliação de intervenções em lugares muito concretos. Os requisitos do desenvolvimento sustentável estabelecem que, além de o projeto ser sustentável em si (Centre de Cultura Contemporánia de Barcelona, 1998), suas formas permitam ou facilitem o comportamento sustentável dos usuários ou cidadãos.

\section{A Agenda 21}

A Agenda 21 tem sua origem na Conferência Rio’92; potencializando-se e concretizando-se em conferências monográficas posteriores. Seu objetivo se centra em instar os municípios e órgãos locais a que elaborem um plano de ação local, como elemento chave para alcançar um desenvolvimento sustentável. A Agenda 21 é uma proposta para a ação inclusiva, participatória e compreensiva (International Council for Local Environmental Initiatives, 2000).

Do ponto de vista da Psicologia Ambiental, existem pelo menos quatro grandes âmbitos de interesse das Agendas 21 em que se pode apresentar contribuições relevantes: 1) A forma da cidade como facilitadora/dificultadora, condicionante e até mesmo determinante de alguns comportamentos dos cidadãos, como é o caso da mobilidade, mas também da criação de atitudes e comportamentos ambientais (City-IdentitySustainability Network, 2002; Gardner \& Stern, 1996; Oskamp, 2000; Ostrom, 1990; Zelezny \& Schultz, 2000); 2) Incidência sobre valores, atitudes e comportamentos, como processo comunicativo e como objeto da educação ambiental (Bator \& Cialdini, 2000; Hernández, Corral, Hess \& Suárez, 2001; Palmer \& Neal, 1994; Pol, Vidal \& Romeo, 2001); 3) A implicação e o compromisso dos cidadãos e as formas de gestão participativa (Castro, 2000; McKenzie-Mohr, 2000; Suárez, 2000) incentivadas pela Agenda 21 ; 4) A criação de indicadores psicosocio-ambientais para o acompanhamento e a avaliação do progresso em direção a um modelo mais sustentável.

\section{Intervenção, gestão e efeito NIMBY}

A tomada de decisões em intervenção e gestão ambientais nem sempre agrada por igual a todos os cidadãos. As administrações públicas devem encontrar lugar para determinados serviços que precisam ser executados, por serem necessários e imprescindíveis, ainda que tenham uma imagem social negativa. É o caso, por exemplo, da gestão de resíduos industriais e do lixo domiciliar. Todo o mundo reconhece a necessidade do serviço, porém ninguém está disposto a ter as instalações necessárias perto de sua casa. Este tipo de rejeição ou resistência ficou conhecido como efeito NIMBY (Not In My Back Yard, não em meu quintal), efeito LULU (Local Unwanted Land Use, uso não desejado do território local), ou efeito BANANA (Building ANything at All Near Anyone, não construir nada perto de ninguém).

O efeito NIMBY pode ser considerado "normal”. Está relacionado à percepção social de risco, à atribuição de causas e potencialidades de alteração do próprio bem-estar e, portanto, levando a condutas de autodefesa (Freudenberg, 1984). Nos parece curioso que, em se tratando de um fenômeno eminentemente ligado à percepção humana, às atitudes e comportamentos dos cidadãos, a problemas de comunicação (ou sua falta) entre gestores e cidadãos, ao desenho urbano 
e de instalações industriais, se encontre tão pouca literatura sobre o tema na Psicologia Ambiental.

A desconfiança na gestão e na tecnologia, além do medo dos efeitos sobre a saúde, são as razoes mais repetidas nos resultados das investigações sobre o efeito NIMBY, bem como outras razões ideológicas e demográficas (e.g., Hunter \& Leyden, 1995). Ainda que o efeito NIMBY possa ter uma expressão individual e grupal, as pessoas que reagem às suas causas não constituem grupos homogêneos (Halstead, Luloff \& Myers, 1993).

Na sociedade moderna há uma superestimativa do risco, tanto nos países desenvolvidos como nos menos desenvolvidos (Dunlap \& Mertig, 1995). Beck (1992a, 1992b) fala de um "novo paradigma da sociedade do risco" diante a necessidade de enfrentar os riscos resultantes da "modernização". As investigações técnicas, sociais e psicológicas constatam que a nocividade e dano dos estímulos ambientais não têm porque ter relação, mesmo que ambas alterem o bem-estar das pessoas. Aspectos como a distância sentida entre uma situação presente e a que se deseja, entre o que se alcançou e o que se considera legitimamente alcançável, resultam muito importantes na avaliação das implicações para o bem-estar. Isso parece intimamente ligado ao locus de controle e a processos de atribuição de causa (Allen \& Ferrand, 1999; Willians \& Less-Haley, 1993), de redução de dissonância cognitiva na concepção de Festinger (1957), ao equilíbrio custo-benefício, à percepção de eqüidade, além do receio de perder os níveis de qualidade de vida alcançados (Lake, 1996; Moreno, Garrido, Martínez, Sandoval \& Saura, 1989/1996). A experiência subjetiva das pessoas aparece como um construto cultural ligado a um sistema de valores que determina uma construção social do que é, e do que não é, mensurável como risco (Valera, 2000).

Os diagnósticos do efeito NIMBY encontrados na literatura permitem planejar estratégias e prever ações possíveis sobre os processos sociais e psicossociais implicados, orientados à administração de tal efeito (ver Pol, 2001, 2003). Certamente, não existem fórmulas mágicas para sua resolução, já que, por definição, o NIMBY sempre surge de uma história própria e se dá em um contexto particular. As soluções ou as estratégias para sua gestão têm de ser construídas sempre de forma específica para cada caso.

O prestígio social das administrações públicas e dos gestores, a informação, a participação e o controle social dos processos tecnológicos e da gestão resultam cruciais para diluir as distorções perceptivas do risco associado, como também para restaurar a confiança e a credibilidade na administração pública.

\section{Para concluir}

Neste trabalho tratamos de perfilar uma serie de âmbitos pouco visíveis na Psicologia Ambiental, mas que constituem parte de uma agenda aplicada que a disciplina está desenvolvendo. A intervenção e a gestão ambientais utilizam os conhecimentos criados a partir da pesquisa básica da Psicologia Ambiental, da Psicologia Social ou da Psicologia em geral, além de outros ramos das ciências sociais, mas também desenvolvem pesquisa e instrumentos próprios. Detivemo-nos em seus aspectos mais específicos para facilitar ao leitor a aproximação a seus conceitos e instrumentos, e nos limitamos a referenciar aqueles aspectos que consideramos estarem ao alcance do leitor na literatura geral da disciplina.

\section{Referências}

Allen, J. B., \& Ferrand, J. L. (1999). Environmental locus of control, sympathy and proenvironmental behavior. A test of Geller's actively caring hypothesis. Environment and Behavior, 31, 338-353.

Allende, J. (1995). Desarrollo Sostenible. De lo global a lo local. Ciudad y Territorio, III(104), 267-281.

Amérigo, M. (2000). Ambientes residenciales. In J. I. Aragonés \& M. Amérigo (Orgs.), Psicología Ambiental (pp. 173-193). Madri: Pirámide.

Bator, R. J., \& Cialdini, R. B. (2000). New ways to promote proenvironmental behavior: the application of persuasion theory to the development of effective proenvironmental public service announcements. Journal of Social Issues, 56(3), 527-541.

Battelle-Columbus Laboratories. (1972). Environmental evaluation system. Springfield, Massachusetts: Autor.

Bechtel, R., \& Churchman, A. (Orgs.). (2002). Handbook of Environmental Psychology ( $2^{\underline{a}}$ ed.). Nova York: Wiley.

Beck, U. (1992a). From industrial society to the risk society: questions of survival social structure and ecological enlightenment. Theory, Culture and Society, 9, 97-123.

Beck, U. (1992b). Risk Society. Towards a new modernity. Londres: Sage.

Bell, P. A., Greene, T. C., Fisher, J. D., \& Baum, A. (1996). Environmental Psychology (4⿳亠口冋 ed.). Fort Worth: Harcourt Brace.

Brundtland, G. H. (1987). Our common future. Oxford: Oxford University Press.

Canter, L. W. (1977/1997). Environmental impact assessment. Nova York: McGraw Hill.

Castro, R. de (2000). Voluntariado ambiental. Claves para la acción proambiental de la comunidad. Illes Ballears: Di7 edicions.

Centre de Cultura Contemporánia de Barcelona. (1998). The sustainable city. Barcelona: Autor.

Cheremisinoff, P. N., \& Cheremisinoff, N. P. (1993). Professional environmental auditor's guidebook. Park Ridge, New Jersey: Noyes.

City-Identity-Sustainability Network Research (2002). Environment and Behavior, 34(1) [Special Issue].

Cobb, J. B. (1995). Toward a just and sustainable economic order. Journal of Social Issues, 51(4), 83-100.

Corson, W. (1995). Priorities for a sustainable future: the role of education, the media and tax reform. Journal of Social Issues, 51(4), 37-61.

Dunlap, R., \& Mertig, A. (1995). Global environmental concern: a challenge to the post-materialism thesis. In P. Ester \& W. Schluter (Orgs.), Social dimensions of contemporary environmental issues: international perspectives. Tilburg: Tilburg University Press.

European Commission. (1985). Council Directive of 27 June 1985 on the assessment of the effects of certain public and private projects on the environment. 85/337/EEC. Official Journal No. L 175, 05/07/1985, pp. 0040-0048. [On-line]. Disponível em: http://europa.eu.int/comm/ environment/eia/full-legal-text/85337.htm

European Commission. (1997). Council Directive 97/11/EC of 3 March 1997 amending Directive 85/337/EEC on the assessment of the effects of certain public and private projects on the environment. Official Journal No. L 073, 14/03/1997, pp. 0005. [On-line]. Disponível em: http://europa.eu.int/comm/ environment/eia/full-legal-text/9711.htm

Festinger, L. (1957). A theory of cognitive dissonance. Stanford, Califórnia: Stanford University Press. 
Fleury-Bahi, G. (1997). Histoire, identité résidentielle et attachement au quartier actuel: étude sur les habitants de la ville de Paris. Psychologie Française, 42(2), 183-184.

Francescato, G., Weidemann, S., \& Anderson, J. R. (1989). Evaluating the built environment from the users' point of view: an attitudinal model of residential satisfaction. In W. F. E. Preiser (Org.), Building evaluation. Nova York: Plenum.

Freudenberg, N. (1984). Not in our backyards: community action for health and the environment. Nova York: Monthly Review Press.

Freudenberg, W. R. (1986). Social impact assessment. Annual Review of Sociology, 12, 451-478.

García-Mira, R., Sabucedo, J. M., \& Romay, J. (Orgs.). (2002). Psicología y medio ambiente. Aspectos psicosociales, educativos y metodológicos. A Coruña: Publiedisa / IAPS.

Gardner, G. T., \& Stern, P. C. (1996). Environmental problems and human behavior. Boston: Allyn \& Bacon.

Halstead, J., Luloff, A., \& Myers, S. (1993). An examination of the NIMBY syndrome: why not in my backyard? Journal of the Community Development Society, 24(1), 88-102.

Harrison, L. (Org.). (1995). Environmental, health and safety auditing handbook ( $2^{\underline{a}}$ ed.). Nova York: McGraw-Hill.

Hernández, B., Corral, V., Hess, S., \& Suárez, E. (2001). Sistemas de creencias ambientales: un análisis multi-muestra de estructuras factoriales. Estudios de Psicología, 22(1), 53-64.

Hillary, R. (1999). Evaluation of study reports on the barriers, opportunities and drivers for SMEs in the adoption of environmental management systems [On-line]. Disponível em: http://www.inem.org/htdocs/iso/ hillary.html\#Anchor-49575

Hunter, S., \& Leyden, K. (1995). NIMBY: explaining opposition to hazardous waste facilities. Policy Studies Journal, 23(4), 601-619.

Huston, M. C., \& DeSouza, G. R. (1980). SIMPCT: a system to forecast impacts of growth and development. In Faculty of Extension, University of Alberta (Org.), Proceedings of the Conference Computer models and forecasting socio-economic impacts of growth and development, Jasper Park Lodge, 20 a 23 de Abril. Edmonton, Alberta, Canadá: Autor.

International Council for Local Environmental Initiatives./ iclei (2000). Local Agenda 21. Model Communities Program. [On-line]. Disponível em: http:www.iclei.org/la21/la21updt.htm

Interorganizational Committee on Guidelines and Principles for Social Impact Assessment / ICGPSIA. (1995). Guidelines and principles for social impact assessment. Environmental Impact Assessment Review, 15, 11-43.

Jørgensen, T. (2000). Environmental management systems and organizational change. Eco-Management and Auditing, 7(2), 60-66.

Kirkland, L. H., \& Thompson, D. (1999). Challenges in designing, implementing and operating an environmental management system. Business Strategy and the Environment, 8(2), 128-143.

Lake, R. (1996). Volunteers, NIMBYs, and environmental justice: dilemmas of democratic practice. Antipode, 28(2), 160-174

Martínez-Alier, J. (1992). De la economía ecológica al ecologismo popular. Barcelona: Icaria.

McKenzie-Mohr, D. (2000). New ways to promote proenvironmental behavior: promoting sustainable behavior: an introduction to community-based social marketing. Journal of Social Issues, 56(3), 543-554.

Milbrath, L. (1986). Environmental beliefs and values. In M. G. Hermann (Org.), Political Psychology: contemporary problems and issues (pp. 97-138). San Francisco: Jossey-Bass.

Milbrath, L. (1995). Psychological, cultural, and informational barriers to sustainability. Journal of Social Issues, 51(4), 101-120.

Moreno, E. (1995). Organization protocol. In J. M. Serena, E. Moreno, J. Pallisé, D. Brugada, J. Ester, G. Herranz \& F. Miret (Orgs.), Environmental Audit Manual. Barcelona: AUMA Environmental Consultancy.
Moreno, E., Garrido, S., Martínez, A., Sandoval, G., \& Saura, C. (1989/1996). Efectos ambientales y rechazo social de una planta de reciclaje de basuras. Propuestas para su minimización [Informe para la Entitat Metropolitana de Serveis Hidraulics i Tractament de Residus] (Monografies Psico/Socio/ Ambientals, 3). Barcelona: Publicacions Universitat de Barcelona.

Moreno, E., \& Pol, E. (1999). Nociones psicosociales para la intervención y la gestión ambiental (Monografies Socio / Ambientals, 14). Barcelona: Publicacions Universitat de Barcelona.

Moreno, E., \& Pol, E. (2002). Metodologies per a la detecció dels impactes sobre el medi social/humà. - Metodologías para la detección de los impactos sobre el medio social/humano - Social/human impact detection methodologies for EIA process [trilíngüe] (Colección Documents dels Quaderns de Medi Ambient, No 8). Barcelona: Generalitat de Catalunya, Dep. Medi Ambient.

Moser, G., Ratiu, E., \& Fleury-Bahi, G. (2002). Appropriation and interpersonal relationships. From dwelling to city through the neighborhood. Environment and Behavior, 34(1), 122-136.

Moxen, J., \& Strachan, P. A. (2000). ISO 14001: a case of cultural myopia. EcoManagement and Auditing, 7(2), 82-90.

Olson, R. (1995). Sustainability as a social vision. Journal of Social Issues, 51(4), 15-35.

Oskamp, S. (2000). Psychological contributions to achieving an ecologically sustainable future for humanity. Journal of Social Issues, 56(3), 373-390.

Ostrom, E. (1990). Governing the commons: the evolution of institutions for collective action. Cambridge, Inglaterra: Cambridge University Press.

Palmer, J., \& Neal, P. (1994). Handbook of Environmental Education. Londres: Routledge.

Pol, E. (1993). Environmental Psychology in Europe. From Architectural Psychology to Green Psychology. Londres: Avebury.

Pol, E. (1996). La apropiación del espacio. In L. Íñiguez \& E. Pol (Orgs.), Cognición, representación y apropiación del espacio (pp. 45-62, Colección Monografias Psico/Socio/Ambientales, 9). Barcelona: Publicacions Universitat de Barcelona.

Pol, E. (1998). Evoluciones de la Psicología Ambiental hacia la sostenibilidad: tres propuestas teóricas y orientaciones para la gestión. In D. Páez \& S. Ayestarán (Orgs.), Los desarrollos de la Psicología Social en España (pp. 105-120). Madri: Infancia y Aprendizaje.

Pol, E. (2001). Impacte social, comunicació ambiental i participació (Monografies Universitàries, No 3). Barcelona: Generalitat de Catalunya, Dep. Medi Ambient.

Pol, E. (2002a). Environmental management: a perspective from Environmental Psychology. In R. B. Bechtel \& A. Churchman (Orgs.), Handbook of Environmental Psychology ( $2^{\underline{a}}$ ed., pp. 55-84). Nova York: Wiley.

Pol, E. (2002b). The theoretical background of the City-Identity-Sustainability (CIS) Network. Environment and Behavior, 34(1), 8-25.

Pol, E. (2003). De l'intervention à la gestion environnementale. In G. Moser \& K. Weiss (Orgs.), Espaces de vie. Aspects de la relation homme-environnement (pp. 305-330). Paris: Armand Colin.

Pol, E. (no prelo). L' appropriation de l' espace. In P. Serfaty-Garzon \& J. Morval (Orgs.), Textes de base de Psychologie Environnementale. Paris: Armand Colin.

Pol, E., \& Moreno, E. (1994). Evaluación del impacto social en los estudios de impacto ambiental. In B. Hernández, J. Martínez \& E. Suárez (Org.), Psicología Ambiental y responsabilidad ecológica (pp. 82-105). Las Palmas de Gran Canaria: Departamento de Psicología y Sociología, Universidad de las Palmas de Gran Canaria.

Pol, E., \& Moreno, E. (2002). DIS/BCN-SID/BCN. Detecció d'impactes socials - Social impact detection. Interactive forms - protocols interactius [CD interativo] (Colección Monografies Socio/Ambientals, 21). Barcelona: Publicacions Universitat de Barcelona.

Pol, E., \& Valera, S. (1999). Symbolisme de l'espace public et identité sociale. Villes en parallèle, 28-29, 13-33. 
Pol, E., Vidal, T., \& Romeo, M. (2001). Supuestos de cambio de actitud y conducta usados en las campañas de publicidad y los programas de promoción ambiental. El modelo de las 4 esferas. Estudios de Psicología, 22(1), 111-126.

Preiser, W. F. E., Rabinowitz, H. Z., \& White, E. T. (1987). Post occupancy evaluation. Nova York: Van Nostrand Reinhold.

Rieradevall, J., Moreno, E., Serena, J. M., \& Pol, E. (1996). Anàlisi del cicle de vida de productes. Millora ambiental de la producció i el consum. In E. Pol \& T. Vidal (Orgs.), Perfils socials en la intervenció ambiental. Una perspectiva professional (pp. 87-101, Colección Monografías Psico/Socio/ Ambientales, 1). Barcelona: Publicacions Universitat de Barcelona.

Schultz, W., \& Schmuck, P. (Orgs.). (2002). The Psychology of sustainable development. Boston: Kluwer.

Stern, P. C., \& Oskamp, S. (1987). Managing scarce environmental resources. In D. Stokols \& I. Altman (Orgs.), Handbook of Environmental Psychology (Vol. 2, pp. 1043-1088). Nova York: Wiley.

Suárez, E. (2000). Problemas ambientales y soluciones conductuales. In J. I. Aragonés \& M. Amérigo (Orgs.), Psicología Ambiental (2ª̣ ed., pp. 331355). Madri: Pirámide.

Taylor, N. C., \& Bryan, H. (1990). A New Zealand issues-oriented approach to social impact assessment. In K. Finsterbusch, J. Ingersoll \& L. Llewellyn
(Orgs.), Methods for social analysis in developing countries (Social impact assessment series, no 17). Boulder, Colorado: Westview Press.

Torgerson, D. (1980). Industrialization and assessment: social impact assessment as a social phenomenon. Toronto: York University.

United States-Environmental Protection Agency. (1969). National Environmental Policy Act (NEPA) [On-line]. Disponível em: http://www.epa.gov/region5/ defs/html/nepa.htm

Valera, S. (2000). La percepción de riesgo en la población. In Diputació de Tarragona (Org.), Fòrum de la Seguretat. La Directiva Seveso II (pp. 240243). Barcelona: Beta.

Valera, S., Íñiguez, L., Pol, E., \& Llueca, J. (1996). Planificació, gestió i qualitat de vida en el medi urbà. In E. Pol \& T. Vidal (Orgs.), Perfils socials en la intervenció ambiental. Una perspectiva professional (pp. 45-55, Colección Monografías Psico/Socio/Ambientales, 1). Barcelona: Publicacions Universitat de Barcelona.

Williams, C. W., \& Less-Haley, P. R. (1993). Perceived toxic exposure: a review of four cognitive influences on perception of illness. Journal of Social Behavior and Personality, 8, 489-506.

Zelezny, L. C., \& Schultz, P. W. (2000). Promoting environmentalism. Journal of Social Issues, 56(3), 365-371.

1 Traduzido de original em espanhol por Milene Pereira, psicóloga e doutoranda em Recursos Humanos e Organizações na Universidade de Barcelona (revisão da tradução por José Q. Pinheiro).

Enric Pol, doutor em Psicologia Social, Universitat de Barcelona, é catedrático de Psicologia Social e Ambiental e Diretor do Máster en Intervención Ambiental: Persona, Sociedad y Gestión, Departamento de Psicologia Social, Universitat de Barcelona. Endereço para correspondência: Departament de Psicologia Social, Universitat de Barcelona, P. Vall d'Hebron 171; 08035, Barcelona. Tel.: +34 933125181. Fax: +34 934021366. E-mail: epol@ub.edu. 